Kwan Yi

Eastern Kentucky University, Richmond, KY, USA

Ping Li

Queens College, City University of New York, Queens, NY, USA

Tao Jin

Louisiana State University, Baton Rouge, LA, USA

\title{
Making Invisible College Visible: Comparing ASIS\&T and CAIS Annual Conferences as Two Scientific Communities (Paper)
}

\begin{abstract}
:
This paper reports an ongoing research that conceptualizes two major LIS academic conferences in North America as two dynamic scientific communities: ASIS\&T Annual Meetings and CAIS Annual Conferences. Using a social network analysis approach, this study compares and visualizes how these two scientific communities have been structured and organized between 1993 and 2015.
\end{abstract}

Résumé:

\section{Introduction}

The notion of scientific community usually refers to a group of scientists and researchers who share their research interests, norms and values in a given field of scientific knowledge domain (Gaillard, 1994; Hagstrom, 1965). A scientific community is then a social institution of science, connecting people with similar research concerns and goals. Its members come and go freely, without needing a decree issued by any authorities. Such loosely formed and ever-changing social institutions are, quite often, invisible, intangible, and amorphous in a way that clear boundaries are difficult to capture (Mulkay et al., 1975; Velden and Lagoze, 2013). Hence, they are also called as invisible colleges (Zuccala, 2006).

Many studies have been done to explore these invisible communities. Rarely, however, an academic conference was considered as a conceptual and observational channel to study properties of scientific communities (Yi, Jin, and Li, 2016). Academic conferences are forums for all scholars and researchers to disseminate research findings, receive feedback, exchange ideas, and get inspirations (Franfurter and Lane, 1993). Such physical gatherings not only provide a social platform and a shared repertoire for participants to communicate and network, but also furnish a visible and tangible opportunity to capture and record the structure and activities of scientific communities.

This paper reports an ongoing research that conceptualizes two major academic conferences on library and information science (LIS) as two dynamic scientific communities: the Association for Information Science and Technology (ASIS\&T) Annual Meetings, and the Canadian Association 
for Information Science/L'Association canadienne des sciences de l'information (CAIS/ACSI) Annual Conferences. Using a social network analysis (SNA) approach, this study explores, visualizes and compares how the two scientific communities have been structured and organized over a designated period of time. The purpose of this research is in three-fold: (1) to capture and examine the social structures of these two communities; (2) to identify any discernable patterns in these structures; and (3) to draw out any similarities and differences between the two communities. With these goals, two research questions guide this research: (1) Within each of the communities, how are its members connected with one another? (2) To what extent, in terms of social structure, are the two scientific communities dissimilar?

\section{Research Context}

Both ASIS\&T and CAIS/ACSI are primary societies on LIS in North America. Prior to 2013, the full name of the ASIS\&T was the American Society for Information Science and Technology, which was updated from the American Society for Information Science (ASIS), an older name used since 1968. ASIS\&T boasts that they are leaders of searching for "new and better theories, techniques, and technologies to improve access to information" for nearly 80 years (ASIS\&T, 2017). While they rapidly increase their international membership recently, a majority of the members come from the United States. Similarly, CAIS/ACSI is a national association fully dedicated to the advancement of information science in Canada. Compared to ASIS\&T, CAIS has a shorter history and smaller membership size.

As aforementioned, few studies have been done to conceptualize academic conferences as scientific communities. Jacob and McFarlane (2005) explored how interactive technologies could be used to enhance knowledge building processes during an academic conference. McKechnie, Julien and Oliphant (2008) examined how scientific research findings would be interpreted and reported for application to a certain group of relevant stakeholders. None of the studies, however, has probed social structures and ordering of scientific communities. On the other hand, in a large published literature on scholarly network, invisible college, and scientific collaboration that focuses on structural problems of scientific communities, academic conferences are seemingly never used as a research lens. An exception is Yi, Jin and Li (2016), who systematically studied the social connections in CAIS/ACSI annual conferences between 1993 and 2015. However, that study concentrated only on one academic conference in one country. We are not sure if similar structural features would be seen when comparing multiple academic conferences. This study attempts to fill that void and develop a perspective.

\section{Methods}

We conduct this study primarily relying on an SNA approach. In this approach, any communities are made up of a set of people or groups socially connected with one another; such social connections can be described in graphical or matrix forms; individual persons or groups are conceptualized as nodes, and the connections or relationships in-between are represented as links or ties (Haythornthwaite, 1996; Newman, 2001a, 2001b, 2001c). Measures of centrality are an essential method in SNA. Here in this study, we adopt three centrality measures: degree centrality, closeness centrality, and betweenness centrality. Degree centrality is measured by counting the number of relationships maintained by each individual in a social network. Closeness centrality focuses on the shortest path in distance between an individual and every other individual in a social network. Betweenness centrality concerns the extent to which an 
individual sits between others in a social network. As stated earlier, we conceptualize annual conferences of ASIS\&T and CAIS/ACSI as scientific communities. Thus, a member of the community is defined as an author who writes and publishes contributed or full paper(s) for such conferences. These members constitute various social networks, which are appropriate to be studied by the SNA approach.

In terms of data collection, we collect our research data (mostly author, bibliographic and affiliation information) from ASIS\&T and CAIS/ACSI conference proceedings (both in print, if applicable, and electronic formats). For a fair comparison, we only collect data about authors of contributed papers (ASIS\&T) or full papers (CAIS/ACSI) that are published between 1993 and 2015. Thus, authors of keynote speeches, panels, or posters are not included in this study. This could be a limitation, but such an approach helps us capture a backbone or core set of members in the communities and, at the same time, build and maintain a manageable size of our data set. The collected data are then normalized to remove varied name spellings and make them standardized for further processing.

In terms of data analysis, our first job is to create an author-to-paper matrix, called publication matrix. It is a publication network in a matrix form that shows the relationship between authors and papers, demonstrating who authors which paper(s). The publication matrix is a binary matrix where each row represents an author and each column represents a paper. Our second job is to construct an author-to-author matrix, called coauthorship matrix. A coauthorship matrix represents a collaboration network, showing how often one author collaborates with another. After the coauthorship matrix is done, the aforementioned measures of centrality come into play. For this study, we use two SNA software applications, UCINET and NetDraw. UCINET is used to measure and calculate those centralities. NetDraw is used to visualize the co-authorship networks, namely, the structures of the scientific communities.

\section{Findings}

First of all, for both conference communities, between 1993 and 2015, large numbers of papers and authors are identified. The ASIS\&T community (1,113 contributed papers published by 1,488 authors) has a larger scale than the CAIS/ACSI community ( 872 full papers published by 777 authors). A set of an estimated 200 authors encompass both communities. Secondly, in ASIS\&T annual meetings, we have more chances to see collaborative papers than in CAIS annual conferences. While two-author papers take a dominant mode in the ASIS\&T community (34\%), single-author papers dominate the CAIS community (50\%). In terms of the average number of authors per paper, ASIS\&T is 2.6, while CAIS/ACSI is 1.8. Thirdly, there is no significant discrepancy between the two communities if we look at the number of co-authors per author: ASIS\&T, 2.97; CAIS/ACSI, 2.26. Fourth, through calculating those centrality metrics, for both communities, we have identified a number of key authors, who are "core actors" in the network and make larger influences than others do. Lastly, the preliminary results show that for both communities, numerous components exist. By the term components, we refer to those separate portions of a community network. For each component, all nodes inside are directly or indirectly connected with one another, but they do not connect with, or are independent from, other portions of the network. The preliminary results obtained from the study show that the ASIS\&T community has nearly 1.5 times more components than what CAIS/ACSI community has (379: 260). 


\section{Concluding Remarks}

As managed events, academic conferences are miniatures of scientific communities. They provide good opportunities to capture the structure of the community and record the activities therein. They can be served as a useful tool to make those invisible colleges visible, so that a deeper perspective and insight about such communities can be obtained. In this research, we analyze and contrast the social structures of ASIS\&T and CAIS/ACSI annual conferences over 23 years. The results show some discernable patterns and identify a few similarities and differences between the two communities. These findings will be useful to help us better understand the dynamics of scientific communities. Also, they will inform community participants and managers on how to better grow and nurture these collaborative networks.

\section{Reference List:}

Association for Information Science and Technology (ASIS\&T). 2017. About ASIS\&T. Retrieved on January 20, 2017 from https://www.asist.org/about/

Frankfurter, G. M., and W. R. Lane. 1993. "The role of annual conferences in academic research, with evidence on the perceived traits of discussants." Financial Practice \&Education, 3(1): 713.

Gaillard, J. 1994. "The behaviour of scientists and scientific communities." In The Uncertain Quest: Science, Technology and Development, edited by Francisco R. Sagasti, Jean Jacques Salomon and Caeline Sachs-Jeantet, 201-236. Tokyo, Japan: United Nations University Press.

Hagstrom, W. 1965. The Scientific Community. New York: Basic Books.

Haythornthwaite, C. 1996. "Social network analysis: An approach and technique for the study if information exchange." Library and Information Science Research, 18: 323-342.

Jacobs, N., and A. McFarlane. 2005. "Conferences as learning communities: Some early lessons in using 'back-channel' technologies at an academic conference - distributed intelligence or divided attention?" Journal of Computer Assisted Learning, 21: 317-329.

McKechnie, L. (E.F.), H. Julien, and T. Oliphant. 2008. "Communicating research findings to library and information science practitioners: a study of ISIC papers from 1996 to 2000.” Information Research, 13(4): paper 375. Retrieved on January 20, 2017 from http://InformationR.net/ir/13-4/paper375.html

Mulkay, M. J., G. N. Gilbert, and S. Woolgar. 1975. "Problem areas and research networks in science." Sociology, 9: 187-203.

Newman, M. E. J. 2001a. "The structure of scientific collaboration networks." Proceedings of the National Academy of Sciences of the United States of America, 98(2): 404-409. 
Newman, M. E. J. 2001b. "Scientific collaboration networks. I. Network construction and fundamental results.” Physical Review, E 64: 016131.

Newman, M. E. J. 2001c. "Scientific collaboration networks, II. Shortest paths, weighted networks, and centrality." Physical Review, E 64: 016132.

Velden, T., and C. Lagoze. 2013. "The extraction of community structures from publication networks to support ethnographic observations of field differences in scientific communication." Journal of the American Society for Information Science and Technology, 64 (12): 2405-2427.

Yi, K., T. Jin, and P. Li. 2016. "Research activities and networks in CAIS Conferences for the period of 1993-2005: Social Network Analysis." Proceedings of the 44th Annual Conference of the Canadian Association of Information Science, University of Calgary, Calgary, Alberta, Canada, June 1-3, 2016.

Zuccala, A. 2006. "Modeling the invisible college." Journal of the American Society for Information Science and Technology, 57(2): 152-168. 